\title{
ESTIMATION FOR DOMAINS IN DOUBLE SAMPLING WITH PROBABILITIES PROPORTIONAL TO KNOWN SIZE
}

\section{GODWIN A. UDOFIA}

(Received 3 July 2002; Revision accepted 5 August $2(132)$

\section{ABSTRACT}

Available publications show that the variance of an estimator of a domain parameter depends on the variance of the study variable for the domain elements and on the variance of the mean of that variable for elements of the domain in each constituent stratum. In this article, we show that the variance of an estimator of a domain tota! for a small domain of study in double sampling with probabilities proportional to size (pps) when the size, $X_{n}$, is known before the start of the survey depends only on the variance of the study variable for elements of the domain within each separate stratum. The reduction in variance of the estimator by knowledge of $X$ is greater when both the proportion of non-domain elements and the deviation of mean of the study variable for the domain elements in each stratum from the population mean for the domain are large while the study variable varies little among the domain elements in each stratum.

Key Words: Domain, Double, Sampling, Unequal, Probabilities

\section{INTRODUCTION}

After sample survey data have been collected totals, means ' $)$ proportions must be estimated. It is usually realised during the analysis of the data that these estii.iates should also refer to certain subpopulations that are also called domains of study. Estimation of domain parameters is straightforward if the domain of study constitutes a separate stratum of the population and no special theory is necessary: see Udofia (2002b). In most cases, a domain of study cuts across constituent strata of the population with unknown number of elements within each stratum.

The problem of estimation for such domains was first considered in detail by Yates (1953) Yates (1953) notes that the variance of an estimator of a domain parameter is increased by the fact that the number of the domain elements, and hence the number of those elements that can fall in a random sample of a fixed size, is unknown before the start of the survey. Durbin (1958) and Hartley (1959) give a derivation of Yates' results in multi-stage sampling. Scott and Smith (1971) extends Yates' results to stratified multistage sampling while Tin and Toe (1972) extends the same results to Bayes estimation for domains in stratified sampling. Udofia (2002a and 2002b) extend Yates' - results to double sampling for pps when information on the size, $X$, of each sampling unit is unknown. Tripathi (1988) gives extension of Yates' results to estimation of proportions in sampling on two occasions using inverse equal probability sampling. The problem of allocation of resources when domains of study are of primary interest is discussed by Kish (1969). Cochran (1977) page 38 shows that knowledge of the size, $N_{i}$, of domain $j$ that is of interest reduces the variance of the estimator of 
domain mean in a single-phase simple random sample design. The reduction in variance is shown to be greater when the proportion of non-domain elements in the population is large and the study variable varies little among the domain elements.

It is well known that stratified ranciom sampling and sampling with pps give a more precise result than equal probability sampling; see U Ch fia (2002b) for reference. It is also of interest therefore to determine the effect of knowledge of the size, $X$, of each sampling unit on the variance of an estimator of a domain total in double sampling using probabilities proportional to size. This article is an attempt to provide a mathematical expression for the variance of an estimator of the domain total in double sampling with pps and to determine the effect of knowledge of $X$ on the variance of such. an estimator.

\section{SAMPLE DESIGN}

The population under study consisting of $\mathrm{N}$ elements is divided into $\mathrm{H}$ strata with $\mathrm{N}_{h}$ elements in stratum $h(h=1,2, \ldots, H)$. Let $D_{h j}$ denote the part of domain $j(j=1,2, \ldots, M)$ in stratum $h$. The number of elements, $N_{h y}$, in $D_{h j}$ is not known. We assume that $0<N_{h j}<N_{h}$. Information on $X$, the size of each element in the population, is known before the start of the survey. For example, in a survey to determine the average household expenditure on consumption items, the household size may be known from records of earlier surveys but whether the household head has the educational qualification required for a particular subclass of the population that is of interest may not be known before the start of the survey. Hence the number of houselnold heads that fall in that particular class remains a random variable.

An initial sample, $S\left(n_{1 h}\right)$, of size $n_{1 h}$ is drawn from stratum $h(h=1,2, \ldots H)$ with probabilities

$p_{i}\left(i=1,2, \ldots, N_{h}\right)$ proportional to $X$ and, for the reason given in Udofia $(2002 b)$, with replacement. We denote by $n_{1 h j}$ the number of units in $S\left(n_{1 n}\right)$ that fall in $D_{h j}$. For a fixed $n_{11}$, the $n_{11 \mathrm{j}}$ units constitute an initial random sample from $D_{h \mathrm{hj}}$, and we assume that $0<n_{1 \mathrm{hj}}<n_{1 \mathrm{~h}}$. A second sample, $S\left(n_{2 h}\right)$, of $n_{2 h}$ units, $n_{2 h}<n_{1 h}$ is drawn from $S\left(n_{1 n}\right)$ by simple random sampling without replacement (SRSWOR) and the study variable, $Y$, is measured on it. We denote by $n_{2 h j}$ the number of units of $S\left(n_{2 n}\right)$ that fall in $D_{h j}$. These $n_{2 h j}$ units constitute a second random sample from $D_{h j}$ and we assume that $0<n_{2 l i j}<n_{2 h}$. We shall refer to the above sample design as double sampling with probability proportional to size. See Raj (1968), page. 145.

ESTIMATOR OF THE TOTAL OF Y FOR DOMAINS

Let,

$$
\begin{aligned}
y_{h i}^{\prime} & =y_{h i j} \quad \text { if the } i^{t h} \text { element is in } D_{h j} \\
& =0 \text { if the } i^{\text {th }} \text { element is not in } D_{h j}
\end{aligned}
$$

Then under the above sample design, an unbiased estimator of the population total of $Y$ for domain $j$ is

$$
\hat{Y}_{j}=\sum_{h=1}^{H} \frac{1}{n_{2 h}} \sum_{i=1}^{n_{2 h}} \frac{y_{h i}^{\prime}}{P_{h i}}
$$


Proof of unbiasedness:

$$
\left.E\left(\hat{Y}_{j}\right)=E_{1}\left\{E_{2}(\hat{Y})_{j}\right)\right\}
$$

From (2)

$$
E_{2}\left(\hat{Y}_{j}\right)=\sum_{h=1}^{H} \frac{1}{n_{2 h}} \sum_{i=1}^{n_{2 n}}\left(\frac{1}{n_{1 h}} \sum_{i=1}^{n_{1 h}} \frac{y_{h i}^{\prime}}{P_{h i}}\right)=\sum_{h=1}^{H} \frac{1}{n_{1 h}} \sum_{i=1}^{n_{1 h}} \frac{y_{h i}^{\prime}}{P_{h i}}
$$

and hence

$$
\begin{aligned}
E\left(\hat{Y}_{j}\right)=E_{1}\left(\sum_{h=1}^{H^{*}} \frac{1}{n_{1 h}} \sum_{i=1}^{n_{1 h}} \frac{y_{h i}^{\prime}}{P_{h i}}\right)= & \sum_{h=1}^{H} \frac{1}{n_{1 h}} \sum_{i=1}^{n_{1 h}}\left(\sum_{i=1}^{N_{h}} P_{h i} \frac{y_{h i}^{\prime}}{P_{h i}}\right) \\
& =\sum_{h=1}^{H} \sum_{j=1}^{N_{h}} y_{h i}^{\prime}
\end{aligned}
$$

Substitution from (1) gives the result.

$$
E\left(\hat{Y}_{j}\right)=\sum_{h=1}^{H} \sum_{i=1}^{N_{h j}} y_{h i j}=\sum_{h=1}^{H} Y_{h j}=Y_{j}
$$

where

$$
Y_{h j}=\sum_{i=1}^{N_{h j}} Y_{h i j}
$$

Sampling Variance of $\hat{Y}_{j}$

The sampling variance of $\hat{Y}_{j}$ is given by the conditional variance formula

$$
V\left(\hat{Y}_{j}\right)=V_{1} E_{2}\left(\hat{Y}_{j}\right)+E_{1} V_{2}\left(\hat{Y}_{j}\right)
$$


From (3),

$$
V_{1} E_{2}\left(\hat{Y}_{j}\right)=V_{1}\left(\sum_{h=1}^{H} \frac{1}{n_{1 h}} \sum_{i=1}^{n_{1 n}} \frac{y_{h i}^{\prime}}{p_{h i}}\right)=\sum_{h=1}^{H} \frac{1}{n_{1 h}^{2}} \sum_{i=1}^{n_{1 h}} V_{1}\left(\frac{y_{h i}^{\prime}}{P_{h i}}\right)
$$

The cross-product terms vanish for ali $h \neq k$ and $i \neq j$ because independence of selection and selection with replacenent in the first plias: of the survey within each stratum.

$$
V_{1} E_{2}\left(\hat{Y}_{j}\right)=\sum_{h=1}^{H} \frac{1}{n_{1 h}} \sum_{i=1}^{N_{h}} P_{h i}\left(\frac{y_{h i}^{\prime}}{P_{h i}}-Y_{h}^{\prime}\right)^{2} ; Y_{h}^{\prime}=\sum_{i=1}^{N_{h}} y_{h i}^{\prime}
$$

Now

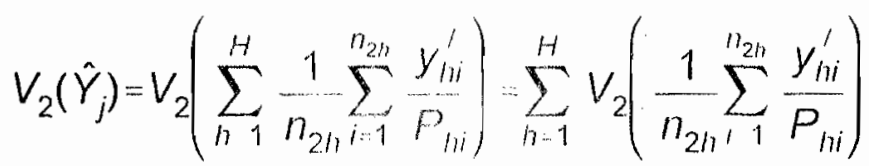

and since selection during the secont phase of the survey was by SRSWol

$$
V_{2}\left(\hat{Y}_{j}\right)=\sum_{h=1}^{H}\left(\frac{1}{n_{2 h}}-\frac{1}{n_{1 h}}\right) \frac{1}{n_{1 h}-1} \sum_{i \cdot 1}^{n_{1 h}}\left(\frac{y_{h i}^{\prime}}{P_{h i}}-\frac{1}{n_{1 h}} \sum_{i=1}^{n_{1 h}} \frac{y_{h i}^{\prime}}{P_{h i}}\right)^{2}
$$

and hence

$$
E V_{2}\left(\hat{Y}_{j}\right)=\sum_{h=1}^{H}\left(\frac{1}{n_{2 h}}-\frac{1}{n_{1 h}}\right) \sum_{i \cdot 1}^{N_{b}} P_{h}\left(\frac{Y_{h i}^{\prime}}{P_{h i}}-Y_{n}^{\prime}\right)^{2}
$$

since

$$
E\left[\frac{1}{n_{1 h}-1} \sum_{i=1}^{n_{1 n}}\left(\frac{y_{h i}^{\prime}}{P_{h i}}-\frac{1}{n_{1 h}} \sum_{i=1}^{n_{1 n}} \frac{y_{h i}^{\prime}}{P_{h i}}\right)^{2}\right]=\sum_{i=1}^{N_{h}} P_{h i}\left(\frac{y_{h i}^{\prime}}{P_{h i}}-Y_{h}^{\prime}\right)^{2}
$$


See Raj (1968), page 146.

Substitution of (5) and (6) in (1) gives the resull:

$$
V\left(\hat{Y}_{j}\right)=\sum_{h=1}^{H} \frac{1}{n_{2 n}} \sum_{i=1}^{N_{h}} P_{h}\left(\frac{y_{h i}^{\prime}}{P_{n i}} y_{h}^{\prime}\right)^{2}
$$

By substituting for $y_{1,1}^{\prime}$ from (1) in (7) we obtain

$$
V\left(\hat{Y}_{j}\right)=\sum_{h=1}^{H} \frac{1}{n_{2 h}} \sum_{i=1}^{N_{l i j}} P_{h n}\left(\frac{y_{i i i}}{P_{m i j}}-Y_{b j}\right)^{2}
$$

where

$$
b_{h i j}=\frac{N_{i i j}}{\sum_{i=1}^{N_{i j}} x_{i i j}}
$$

is the probability that the it elemont falls in D.

\section{ESTIMATION OF $V\left(\hat{Y}_{j}\right)$}

Since the values of $Y$ for the domain are available only for the subsample of $n_{2 h \mathrm{j}}$ units of $S\left(n_{2 h}\right)$ and

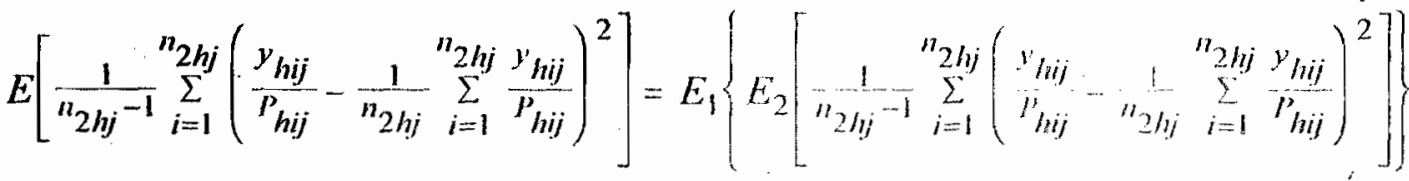

$$
\begin{aligned}
& =E_{1}\left[\frac{1}{n_{1 h i j}-1} \sum_{i=1}^{n_{1 h j}}\left(\frac{y_{h i j}}{P_{h i j}}-\frac{1}{n_{1}{ }_{i j}} \sum_{i=1}^{n_{1 h i}} \frac{y_{h i j}}{P_{h i j}}\right)^{2}\right] \\
& =\sum_{i=1}^{N_{h i j}} P_{h i j}\left(\frac{y_{h i j}}{P_{h i j}}-Y_{h i}\right)^{2}
\end{aligned}
$$


an unbiased estimator of $\mathrm{V}\left(\hat{\hat{Y}}_{j}\right)$ in $(s)$ is given hy

$$
\hat{V}\left(\hat{Y}_{j}\right)=\sum_{h=1}^{H} \frac{1}{n_{2 h}\left(n_{2 h j}-1\right)} \sum_{i=1}^{n_{2 h j}}\left(\frac{y_{h i j}}{P_{h i j}}-\frac{1}{n_{2 h j}} \cdot \sum_{i=1}^{n_{2 h i}} \frac{y_{h i j}}{P_{h i j}}\right)^{2}
$$

\section{COMPARISON OFV $\left(\hat{Y}_{j}\right)$ WITH VARIANCE OF A CORRESPONDING ESTIMATOR IN DOUBLE SAMPLING FOR PPS}

When information on the size, $X$, of each eloment of the population is not available before the start nt? the survey, the double sample strategy for estimation of domain total is given by Udofia (2000b) and is called double sampling for pps, see also Raj (1968) page 142. The variance of the estimator of the domain total $n$ double sampling for pps is given by Udofia (2002b) as

$$
V\left(\hat{Y}_{j}\right)=\sum_{h=1}^{l l} \frac{N_{h}}{N_{h}-1} \frac{n_{1 h}-1}{n_{2 h} h_{1 h}} V_{p}(y)_{h j}+\sum_{h=1}^{L} \frac{N_{h}\left(N_{h}-n_{1 h}\right)}{\left(N_{h}-1\right) n_{1 h}}\left\{\left(N_{h j}-1\right) S_{y(h j)}^{2}+N_{h i}\left(1-\frac{n_{h j}}{N_{h}}\right)\left(Y_{h j} \cdots \bar{Y}_{j}\right)^{2}\right\}
$$

Eor large $\mathrm{N}_{\mathrm{h}}$, this can be writlitis ds

$$
V\left(\hat{Y}_{j}\right)=\sum_{h=j}^{H} \frac{n_{1 h}-1}{n_{2 h} n_{1 h}} V_{p}(y)_{h j}+\sum_{h=1}^{H} \frac{1}{n_{1 h}} N_{h}^{2} P_{h j}\left[S_{y(h j)}^{2}+Q_{h j}\left(\bar{Y}_{h j}-\bar{Y}_{j}\right)^{2}\right]
$$

where $P_{h j}=N_{h j} / N_{h}$ is the proportion of elements of domain $j$ in stratum $h$ and $Q_{h j}=1-P_{h j}$ is the proportion of non-domain j elements in stratum $h$. We note that equation (8) can be written as

$$
V\left(\hat{Y}_{j}\right)=\sum_{n+1}^{H} \frac{1}{n_{2 h}} V_{p}(y)_{h j}
$$

where

$$
V_{p}(y)_{h j}=\sum_{i=k} x_{n i} n\left(\frac{y_{h i j}}{x_{h i j}}-\frac{y_{n k j}}{x_{h k j}}\right)^{2}
$$

corresponds to the same expression in equation 9, see Raj (1968), page. 133.

Whereas, when $X$ is known, the variance of $\hat{Y}_{j}$ as given in (8) depends only on components of variance of the study variable for elements of the domain in each constituent stratum of the population, when 
$X$ is unknown the variance of $\hat{Y}_{i}$, as given in equation (9), depends on both the variability of the study variable for elements of the domain in each stratum: and the variability of the mean of the study variable for subsets of the domain elements among the constituent strata. It is important to determine the major factors that lead to the reduction in variance by a knowledge of $X$.

Let $\mathrm{V}_{1 p}\left(\hat{Y}_{j}\right)$ denote the variance in $(9)$ and $\mathrm{V}_{2 p} \hat{Y}_{j}$ the variance ili (10). Then following the approach in Cochran (1977) page 38 ,

$$
\begin{aligned}
& \frac{V_{2 p}\left(\hat{Y}_{j}\right)}{V_{1 p}\left(\hat{Y}_{j}\right)}=\frac{1}{\sum_{h=1}^{H}\left(1-\frac{1}{n_{1 h}}\right)+\sum_{h=1}^{H}\left(N_{h} N_{h j} n_{2 h} / n_{1 h}\right)\left[1+Q_{h j}\left(\bar{Y}_{h j}-\bar{Y}_{j}\right)^{2} / V_{p}(y)_{h j}\right]} \\
& \frac{V_{2 p}\left(\hat{Y}_{j}\right)}{V_{1 p}\left(\hat{Y}_{j}\right)}=\frac{1}{\sum_{h=1}^{H}\left(1-\frac{1}{n_{1 h}}\right)+\sum_{h=1}^{H} N_{h}^{2} W_{2 h} P_{h j}\left[1+Q_{h j}\left(\bar{Y}_{h j}-\bar{Y}_{j}\right)^{2} / V_{p}(y)_{h j}\right]}
\end{aligned}
$$

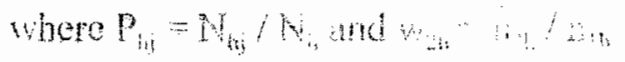

REMARK

The estimator of $\hat{Y}_{j}$ in clouble sampling with probability proportional to size when $\mathrm{X}$ is known is more precise than the corresponding estimator in double sampling for probability proportoional to size when $X$ is not known. Equation (9) has all the attributes proposed by Yates (1953) and proved by all the other results quoted above. Equation (8) is different from all these other results only in the sense that it is not affected by variability of the mean of the study variable for subsets of the domain elements in the different strata. When $P_{h j}=0$, the domain coincides with a stratum and the reduction in variance of the estimator due to knowledge of $X$ is equal to $\sum_{h=1}^{H}\left(1-\frac{1}{n_{1 h}}\right)$. which is the same in global estimation. In this case, no new theory is necessery as earlier concluded by Udofia (2002b).

In equation (11), $\mathbb{N}_{h}, n_{h \mathrm{~h}}$ and $\mathrm{W}_{2 \mathrm{~h}}$ are fixed numbers, By our assumption $Q \leqslant Q_{h j} \leqslant 1$. The reduction in the variance of $\hat{Y}_{j}$ by knowledge of $X$ and hence double sampling with probability proportional to $X$ is greater when both $\mathrm{Q}_{\mathrm{hi}}$, the proportion of nen=domain clements in stratum $h(h-1,2, \ldots, H)$, and $\bar{Y}_{h i}=\bar{Y}_{j}$ (the deviation of the mean of the study variable for $D_{h j}$ from the population mean of the study variable for the domain) are large while $V_{\beta}(y)_{\text {hij }}$, the variance of the study variable for the subclass 
$D_{\mathrm{hj}}$ is small. Since strata are usually formed to be internally homogeneous and externally heterogeneous, $V_{p}$ $(y)_{h j}$ and hence $V_{2 p}\left(\hat{Y}_{j}\right)$ will always be small and

$\bar{Y}_{h j}-\bar{Y}_{j}$ will be large. The magnitude of the reciuction in the variance of $\hat{Y}_{j}$ by knowledge of $X$ therefore depends on efficiency of the stratification procedtre

\section{REFERENCES}

Cochran, W. G., 1977. Sampling Techniques. John Wiley and Sons Inc., New York.

Durbin, J., 1958. Sampling Theory for estinates, based on fewer individuals than the nunber selected. Bull. Int. Stat, Inst, 361. 113-119.

Hartley, H.O., 1959. Analytical Studies or Survey Data. Instituto di Statislica. Rome.

Kish, L., 1969. Design and estimation for subclasses, comparisons and analytical statistics, in, Johnson, N. L. and Smith, and Illar:" Jr eds., (Editors), New Developments in Survey Sampling. Julm Wiley and Sons lne., New York.

Raj, D., 1968. Sampling Theory. McGraw Hill Book Co., New York.

Scott, A. and Smith, T.M.F., 1971. Bayes estimates for subclasses in stratified sampling. Jour. Amer. Stal. Assoc., $60: 8.44$.

Tin, M. and Toe, T., 1972. Estimation for domains inmultistage sampling. Jour. Ancer. Stat. Assoc, 67:913-916.

Tripathi, T. P., 1988. Estimation for domains in sanupling un two occasions. Salnkhya, 50: $103-110$.

Udofia, G., 2002a. Estimation for donains in double sampling for probabilities proportional to size. Sankliya, B, in primt.

Udolia, G., 2002b. On the precision of an estimator of mean for domains in touble sampling for inclusion probabilities. Ghutal Journ: of Pure and Appl. Sciences, $8(4)$ in print:

Yates, F., 1953. Sampling Methods for Censuses and Surveys. Charles Griflin and Co., London. 